\title{
A Note on Harmonious Coloring of Caterpillars
}

\author{
Asahi TAKAOKA ${ }^{\dagger a)}$, Member, Shingo OKUMA ${ }^{\dagger}$, Nonmember, Satoshi TAYU ${ }^{\dagger b)}$, Member, \\ and Shuichi UENO ${ }^{\dagger c}$, Fellow
}

\begin{abstract}
SUMMARY The harmonious coloring of an undirected simple graph is a vertex coloring such that adjacent vertices are assigned different colors and each pair of colors appears together on at most one edge. The harmonious chromatic number of a graph is the least number of colors used in such a coloring. The harmonious chromatic number of a path is known, whereas the problem to find the harmonious chromatic number is NP-hard even for trees with pathwidth at most 2 . Hence, we consider the harmonious coloring of trees with pathwidth 1 , which are also known as caterpillars. This paper shows the harmonious chromatic number of a caterpillar with at most one vertex of degree more than 2 . We also show the upper bound of the harmonious chromatic number of a 3-regular caterpillar. key words: caterpillars, Eulerian trail, harmonious coloring, harmonious chromatic number, pathwidth
\end{abstract}

\section{Introduction}

A proper coloring of an undirected simple graph $G$ is an assignment of colors (or numbers) to the vertices of $G$ such that adjacent vertices are assigned different colors. A harmonious coloring of a graph is a proper vertex coloring such that each pair of colors appears together on at most one edge. The harmonious chromatic number $h(G)$ of a graph $G$ is the least number of colors used in such a coloring of $G$. The harmonious coloring problem is to find $h(G)$ of a graph $G$.

The harmonious coloring [13], [17], [18] was developed from the closely related concept of line-distinguishing coloring [12], [14], and has been studied in the literature (e.g. [10], [16] for surveys and [1], [2], [8] for recent results). The harmonious coloring has potential applications to minimal perfect hash functions [9] and aviation guidance systems [16]. The harmonious coloring problem is very difficult in general, and it is known to be NP-hard for several restricted classes of graphs [3]-[5], [7], [11], [14], [15].

The harmonious chromatic number of a path is known [14], [17], whereas the problem is NP-hard even for trees with pathwidth at most 2 [11]. Hence, we consider the harmonious coloring of trees with pathwidth 1 , which are also known as caterpillars. A caterpillar is a tree that has a central path such that every vertex of the tree is on the path

Manuscript received March 30, 2015.

Manuscript revised June 29, 2015.

Manuscript publicized August 28, 2015.

${ }^{\dagger}$ The authors are with the Department of Communications and Computer Engineering, Tokyo Institute of Technology, Tokyo, 152-8550 Japan.

a)E-mail: asahi@eda.ce.titech.ac.jp

b)E-mail: tayu@eda.ce.titech.ac.jp

c) E-mail: ueno@eda.ce.titech.ac.jp

DOI: $10.1587 /$ transinf.2015EDP7113

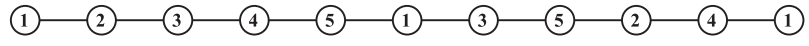

Fig. 1 A harmonious coloring of path $P_{11}$ with 5 colors.

or adjacent to a vertex on the path.

This paper shows the harmonious chromatic number of a caterpillar with at most one vertex of degree more than 2 . The class of such caterpillars can be partitioned into four classes, the class of paths, stars, shooting stars, and comets. For each class, we show the harmonious chromatic number of a caterpillar in the class. In addition, we show the upper bound of the harmonious chromatic number of a 3-regular caterpillar.

The paper is organized as follows. Section 2 describes our main results. Sections 3 to 6 are devoted to the proofs. We conclude with some discussions and remarks in Sect. 7.

\section{Harmonious Coloring of Caterpillars}

In the rest of the paper, let $V(G)$ and $E(G)$ denote the set of vertices and edges of a graph $G$, respectively. Also, let $n=$ $|V(G)|$ and $m=|E(G)|$. If a graph $G$ can be harmoniously colored with $k$ colors, then $m \leq\left(\begin{array}{l}k \\ 2\end{array}\right)$. Let $k(G)$ be the smallest integer $k$ fulfilling this inequality. We can express $k(G)$ as a function of $m$, namely $k(G)=\lceil(1+\sqrt{8 m+1}) / 2\rceil$. Since every graph can be colored harmoniously with $n$ colors, we observe that for any graph $G, k(G) \leq h(G) \leq n$.

\subsection{Paths and Stars}

Let $P_{t}=\left(v_{1}, v_{2}, \ldots, v_{t}\right)$ be a path on $t$ vertices. The harmonious coloring of a path with $k$ colors is obtained from a trail traversing edges of a complete graph $K_{k}$ [14], [17]. The vertices on the path can be colored harmoniously according to the trail.

\section{Theorem A.}

$$
h\left(P_{t}\right)= \begin{cases}k & \text { if } k \text { is odd, } \\
k & \text { if } k \text { is even and } m \leq\left(\begin{array}{l}
k \\
2
\end{array}\right)-\frac{k}{2}+1, \\
k+1 & \text { otherwise, }\end{cases}
$$

where $k=k\left(P_{t}\right)$.

For example, path $P_{11}$ in Fig. 1 can be colored harmoniously with 5 colors according to the Eulerian trail of $K_{5}$.

A complete bipartite graph $K_{1, \Delta}(\Delta \geq 1)$ is called a 
star. The following theorem shows the harmonious chromatic number of a star [10], [16]. The theorem is trivial since the harmonious coloring of stars has to assign a unique color to each vertex.

Theorem B. $h\left(K_{1, \Delta}\right)=\Delta+1$.

\subsection{Shooting Stars and Comets}

Let $P_{t}=\left(v_{1}, v_{2}, \ldots, v_{t}\right)$ be a path on $t$ vertices. A shooting star is a caterpillar obtained from a path $P_{t}(t \geq 4)$ and a star $K_{1, \Delta}(\Delta \geq 1)$ by identifying a vertex in $\left\{v_{2}, v_{t-1}\right\}$ of $P_{t}$ with the degree- $\Delta$ vertex of $K_{1, \Delta}$. A comet is a caterpillar obtained from $P_{t}(t \geq 5)$ and $K_{1, \Delta}(\Delta \geq 1)$ by identifying a vertex in $\left\{v_{3}, v_{4}, \ldots, v_{t-2}\right\}$ of $P_{t}$ with the degree- $\Delta$ vertex of $K_{1, \Delta}$. In other words, a shooting star is a caterpillar obtained from $K_{1, \Delta}(\Delta \geq 3)$ by replacing an edge with a path of length at least 2 , and a comet is a caterpillar obtained from $K_{1, \Delta}(\Delta \geq$ 3 ) by replacing two edges with paths of length at least 2 . We denote by $S_{t, \Delta}(\Delta \geq 3)$ the shooting star with the longest path on $t$ vertices and the maximum degree $\Delta$, and we denote by $C_{t, \Delta}(\Delta \geq 3)$ a comet with the longest path on $t$ vertices and the maximum degree $\Delta$. Notice that a shooting star is uniquely determined by $t$ and $\Delta$, whereas a comet is not. Examples of shooting star and comet are shown in Figs. 2 (a) and $2(\mathrm{~b})$.

We can see that the class of caterpillars with at most one vertex of degree more than 2 can be partitioned into four classes, the class of paths, stars, shooting stars, and comets. The harmonious chromatic number of a path and a star is shown in Theorems A and B, respectively. In the following, we show the harmonious chromatic number of a shooting star and a comet, which we prove in Sects. 4 and 5, respectively.

The harmonious chromatic number of a shooting star is shown by Marszakowska (as cited in [16, Theorem 7.10]). We give here a simpler version of the theorem.

\section{Theorem 1.}

$$
h\left(S_{t, \Delta}\right)= \begin{cases}\Delta+1 & \text { if } \Delta \geq h, \\
h & \text { if } \Delta<h, \text { and } \\
& h \text { is odd and } m \leq\left(\begin{array}{l}
h \\
2
\end{array}\right)-\left\lfloor\frac{\Delta-1}{2}\right\rfloor, \text { or } \\
& \quad h \text { is even and } m \leq\left(\begin{array}{l}
h \\
2
\end{array}\right)-\left\lfloor\frac{h-\Delta}{2}\right\rfloor, \\
h+1 & \text { otherwise. }\end{cases}
$$

where $h=h\left(P_{t}\right)$.

We also show the harmonious chromatic number of a comet. Although the comet $C_{t, \Delta}$ is not uniquely determined by $t$ and $\Delta$, we can express $h\left(C_{t, \Delta}\right)$ only with $t$ and $\Delta$.

\section{Theorem 2.}

$$
h\left(C_{t, \Delta}\right)= \begin{cases}\Delta+1 & \text { if } \Delta \geq h, \\
h & \text { if } \Delta<h, \text { and } \\
& h \text { is odd and } m \leq\left(\begin{array}{l}
h \\
2
\end{array}\right)-\left\lfloor\frac{\Delta-3}{2}\right\rfloor, \text { or } \\
& \quad \text { his even and } m \leq\left(\begin{array}{l}
h \\
2
\end{array}\right)-\left\lfloor\frac{h-\Delta}{2}\right\rfloor, \\
h+1 & \text { otherwise. }\end{cases}
$$

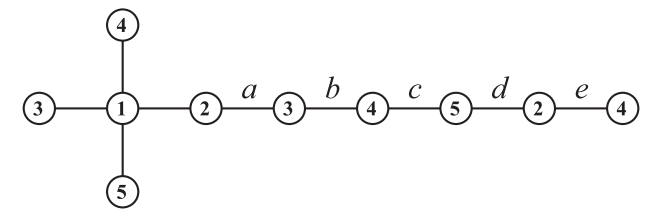

(a) A harmonious coloring of shooting star $S_{8,4}$ with 5 colors.

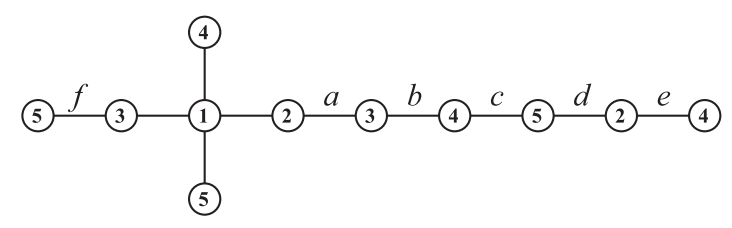

(b) A harmonious coloring of comet $C_{9,4}$ with 5 colors.

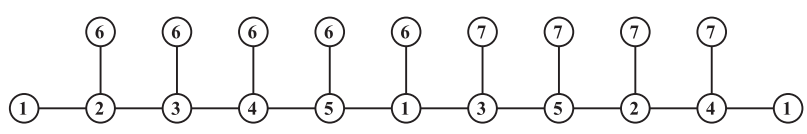

(c) A harmonious coloring of 3-regular caterpillar $T_{11}$ with 7 colors.

Fig. 2 Examples of shooting star, comet, and 3-regular caterpillar.

where $h=h\left(P_{t}\right)$.

The difference between the harmonious chromatic number of shooting star $S_{t, \Delta}$ and comet $C_{t, \Delta}$ arises only when $\Delta<h\left(P_{t}\right)$ and $h\left(P_{t}\right)$ is odd. For example, comet $C_{9,4}$ can be colored harmoniously with $h\left(P_{9}\right)=5$ colors as shown in Fig. 2 (b), since $\left|E\left(C_{9,4}\right)\right| \leq\left(\begin{array}{l}5 \\ 2\end{array}\right)-\left\lfloor\frac{4-3}{2}\right\rfloor=10$. On the other hand, shooting star $S_{9,4}$ cannot be colored harmoniously with 5 colors, since $\left|E\left(S_{9,4}\right)\right|>\left(\begin{array}{l}5 \\ 2\end{array}\right)-\left\lfloor\frac{4-1}{2}\right\rfloor=9$. We note that $S_{8,4}$ can be colored harmoniously with 5 colors as shown in Fig. 2 (a).

\subsection{Three-Regular Caterpillars}

A caterpillar is said to be 3-regular if every inner vertex (vertex with degree more than 1) has degree 3 . In other words, a 3-regular caterpillar is obtained from a path by adding a degree- 1 vertex to each inner vertex. An example of 3-regular caterpillar is shown in Fig. 2 (c). Let $T_{t}$ be a 3-regular caterpillar with the longest path on $t$ vertices, and let $P_{t}$ be the longest path of $T_{t}$. We have the upper bound of the harmonious chromatic number of a 3-regular caterpillar, which we prove in Sect. 6 .

Theorem 3. $h\left(T_{t}\right) \leq h+\lfloor(h-1) / 2\rfloor$, where $h=h\left(P_{t}\right)$.

\section{Preliminaries}

Let $K_{h}$ be a complete graph with $V\left(K_{h}\right)=\left\{v_{1}, v_{2}, \ldots, v_{h}\right\}$. We assume that each $v_{i}$ has color $i$. We consider the graph $K_{h}^{\Delta}(3 \leq \Delta<h)$ obtained from $K_{h}$ by deleting edges $\left(v_{1}, v_{2}\right),\left(v_{1}, v_{3}\right), \ldots,\left(v_{1}, v_{\Delta+1}\right)$. See Fig. 3 for example. Trivially, we have $\left|E\left(K_{h}^{\Delta}\right)\right|=\left(\begin{array}{l}h \\ 2\end{array}\right)-\Delta$. We define two subsets of $V\left(K_{h}^{\Delta}\right)$ as follows:

$$
A\left(K_{h}^{\Delta}\right)=\left\{v_{2}, v_{3}, \ldots, v_{\Delta+1}\right\} ;
$$




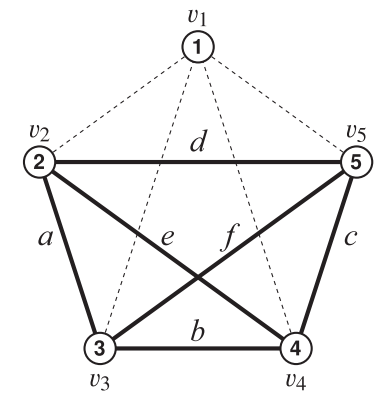

Fig. 3 Graph $K_{5}^{4}$. Dotted lines denote the edges deleted from $K_{5}$.

$$
B\left(K_{h}^{\Delta}\right)=\left\{v_{\Delta+2}, v_{\Delta+3}, \ldots, v_{h}\right\}
$$

If $K_{h}^{\Delta}$ is clear from the context, we denote them by $A$ and $B$ instead of $A\left(K_{h}^{\Delta}\right)$ and $B\left(K_{h}^{\Delta}\right)$, respectively. Notice that sometimes $B=\emptyset$ as $K_{5}^{4}$ in Fig. 3 .

A vertex with odd degree is called an odd vertex, and a vertex with even degree is called an even vertex. We have that $|A|=\Delta$ and $|B|=h-\Delta-1$. The degree of $v_{1}$ is $h-\Delta-1$, the degree of vertices in $A$ is $h-2$, and the degree of vertices in $B$ is $h-1$. Hence, we have the following:

- If $h$ is odd and $\Delta$ is odd, then the degree of $v_{1}$ is odd, the degree of vertices in $A$ is odd, and the degree of vertices in $B$ is even, and hence, $K_{h}^{\Delta}$ has $\Delta+1$ odd vertices;

- If $h$ is odd and $\Delta$ is even, then the degree of $v_{1}$ is even, the degree of vertices in $A$ is odd, and the degree of vertices in $B$ is even, and hence, $K_{h}^{\Delta}$ has $\Delta$ odd vertices;

- If $h$ is even and $\Delta$ is odd, then the degree of $v_{1}$ is even, the degree of vertices in $A$ is even, and the degree of vertices in $B$ is odd, and hence, $K_{h}^{\Delta}$ has $h-\Delta-1$ odd vertices;

- If $h$ is even and $\Delta$ is even, then the degree of $v_{1}$ is odd, the degree of vertices in $A$ is even, and the degree of vertices in $B$ is odd, and hence, $K_{h}^{\Delta}$ has $h-\Delta$ odd vertices.

A trail of a graph is called Eulerian if it traverses every edge of the graph. The following is a well-known fact. The proof can be found in standard textbooks on graph theory [6].

Theorem C. A connected graph $G$ has an Eulerian trail if and only if $G$ has zero or two odd vertices. Moreover, if $G$ has two odd vertices, all Eulerian trails start at one of them and end at the other. If $G$ has no odd vertices, all Eulerian trails are closed.

\section{Proof of Theorem 1}

We prove Theorem 1 by a series of lemmas. Let $P_{t}$ be the longest path of $S_{t, \Delta}$, and let $h=h\left(P_{t}\right)$. The following indicates the first case of Theorem 1 .

Lemma 4. If $\Delta \geq h$, then $h\left(S_{t, \Delta}\right)=\Delta+1$.

Proof. Since we have from Theorem B that $h\left(S_{t, \Delta}\right) \geq \Delta+1$, it suffices to show that $S_{t, \Delta}$ can be colored harmoniously with $\Delta+1$ colors if $\Delta \geq h$. First, we color the vertices on $P_{t}$ harmoniously with colors $1,2, \ldots, h$. Next, we recolor the degree- $\Delta$ vertex with color $\Delta+1$ (Recall that $\Delta+1>h)$. Finally, we color the remaining vertices, that is, the vertices adjacent to the degree- $\Delta$ vertex, with colors $1,2, \ldots, \Delta$. It is straightforward to see that $S_{t, \Delta}$ is colored harmoniously.

Similarly, we have the following.

Lemma 5. If $\Delta<h$, then $h\left(S_{t, \Delta}\right) \leq h+1$.

Proof. We show that $S_{t, \Delta}$ can be colored harmoniously with $h+1$ colors if $\Delta<h$. First, we color the vertices on $P_{t}$ harmoniously with colors $1,2, \ldots, h$. Next, we recolor the degree- $\Delta$ vertex with color $h+1$. Finally, we color the remaining vertices with colors $1,2, \ldots, \Delta$ (Recall that $\Delta<h+1)$. It is straightforward to see that $S_{t, \Delta}$ is colored harmoniously.

Since $P_{t}$ is a subgraph of $S_{t, \Delta}$, we have the following.

Lemma 6. $h\left(S_{t, \Delta}\right) \geq h$.

The following lemma completes the proof of Theorem 1. The rest of the section is devoted to the proof of the lemma.

Lemma 7. Suppose that $\Delta<h . S_{t, \Delta}$ can be colored harmoniously with $h$ colors if and only if

- $m \leq\left(\begin{array}{l}h \\ 2\end{array}\right)-\frac{\Delta-1}{2}$ when $h$ is odd and $\Delta$ is odd,

- $m \leq\left(\begin{array}{l}h \\ 2\end{array}\right)-\frac{\Delta-2}{2}$ when $h$ is odd and $\Delta$ is even,

- $m \leq\left(\begin{array}{l}h \\ 2\end{array}\right)-\frac{h-\Delta-1}{2}$ when $h$ is even and $\Delta$ is odd, and

- $m \leq\left(\begin{array}{l}h \\ 2\end{array}\right)-\frac{h-\Delta}{2}$ when $h$ is even and $\Delta$ is even.

Proof. We assume w.l.o.g. that color 1 is assigned to the degree- $\Delta$ vertex of $S_{t, \Delta}$, and colors $2,3, \ldots, \Delta+1$ are assigned to the vertices adjacent to it (Recall that $\Delta+1 \leq h$ ). See Fig. 2 (a) for example. Then, $t-3$ vertices remains uncolored, and they induce the path $P_{t-3}$ of $S_{t, \Delta}$. To prove Lemma 7, it suffices to show that the remaining path can be colored harmoniously with $h$ colors if and only if the number of edges of $S_{t, \Delta}$ satisfies the inequality in the lemma. We assume without loss of generality that the end-vertex of the path is adjacent to the vertex with color 2 (See Fig. 2 (a)).

We use the graph $K_{h}^{\Delta}$ defined in Sect. 3. Recall that $V\left(K_{h}^{\Delta}\right)=\left\{v_{1}, v_{2}, \ldots, v_{h}\right\}$ and that each $v_{i}$ has color $i$. Notice that the edges of $K_{h}^{\Delta}$ denote the pair of colors which we can use for the harmonious coloring of the remaining path, and the non-edges of $K_{h}^{\Delta}$ denote the pair of colors used for the harmonious coloring of the star $K_{1, \Delta}$ in $S_{t, \Delta}$. Coloring the remaining path of $S_{t, \Delta}$ harmoniously is equivalent to obtaining a trail of $K_{h}^{\Delta}$ with length $t-3$ starting at $v_{2}$. For example, $K_{5}^{4}$ in Fig. 3 has trail $\left(v_{2}, a, v_{3}, b, v_{4}, c, v_{5}, d, v_{2}, e, v_{4}\right)$ of length 5 . As shown in Fig. 2 (a), the remaining path of $S_{8,4}$ can be colored harmoniously according to the trail. Then, the following claim proves Lemma 7 (Recall that $\left|E\left(K_{h}^{\Delta}\right)\right|=\left(\begin{array}{l}h \\ 2\end{array}\right)-\Delta$ ). 
Claim 8. $K_{h}^{\Delta}$ has a trail starting at $v_{2}$ if and only if the length of the trail is at most

- $\left(\begin{array}{l}h \\ 2\end{array}\right)-\Delta-\frac{\Delta-1}{2}$ when $h$ is odd and $\Delta$ is odd,

- $\left(\begin{array}{l}h \\ 2\end{array}\right)-\Delta-\frac{\Delta-2}{2}$ when $h$ is odd and $\Delta$ is even,

- $\left(\begin{array}{l}h \\ 2\end{array}\right)-\Delta-\frac{h-\Delta-1}{2}$ when $h$ is even and $\Delta$ is odd, and

- $\left(\begin{array}{l}h \\ 2\end{array}\right)-\Delta-\frac{h-\Delta}{2}$ when $h$ is even and $\Delta$ is even.

Proof of the "if" part of Claim 8. We distinguish four cases with respect to the parity of $h$ and $\Delta$.

Case $1 h$ is odd and $\Delta$ is odd: Let $G_{h}^{\Delta}$ be the subgraph of $K_{h}^{\Delta}$ obtained by deleting edges $\left(v_{3}, v_{4}\right),\left(v_{5}, v_{6}\right), \ldots$, $\left(v_{\Delta}, v_{\Delta+1}\right)$. Since every vertex in $V\left(G_{h}^{\Delta}\right) \backslash\left\{v_{1}, v_{2}\right\}$ has even degree, $G_{h}^{\Delta}$ has an Eulerian trail from $v_{2}$ to $v_{1}$. Hence, $K_{h}^{\Delta}$ has the trail of length $\left(\begin{array}{l}h \\ 2\end{array}\right)-\Delta-\frac{\Delta-1}{2}$.

Case $2 h$ is odd and $\Delta$ is even: Let $G_{h}^{\Delta}$ be the subgraph of $K_{h}^{\Delta}$ obtained by deleting edges $\left(v_{4}, v_{5}\right),\left(v_{6}, v_{7}\right), \ldots$, $\left(v_{\Delta}, v_{\Delta+1}\right)$. Since every vertex in $V\left(G_{h}^{\Delta}\right) \backslash\left\{v_{2}, v_{3}\right\}$ has even degree, $G_{h}^{\Delta}$ has an Eulerian trail from $v_{2}$ to $v_{3}$. Hence, $K_{h}^{\Delta}$ has the trail of length $\left(\begin{array}{l}h \\ 2\end{array}\right)-\Delta-\frac{\Delta-2}{2}$.

Case $3 h$ is even and $\Delta$ is odd: Let $G_{h}^{\Delta}$ be the subgraph of $K_{h}^{\Delta}$ obtained by deleting edges $\left(v_{\Delta+2}, v_{\Delta+3}\right),\left(v_{\Delta+4}, v_{\Delta+5}\right)$, $\ldots,\left(v_{h-1}, v_{h}\right)$. Since every vertex of $G_{h}^{\Delta}$ has even degree, $G_{h}^{\Delta}$ has an Eulerian closed trail. Hence, $K_{h}^{\Delta}$ has the trail of length $\left(\begin{array}{l}h \\ 2\end{array}\right)-\Delta-\frac{h-\Delta-1}{2}$.

Case $4 h$ is even and $\Delta$ is even: Let $G_{h}^{\Delta}$ be the subgraph of $K_{h}^{\Delta}$ obtained by deleting edges $\left(v_{\Delta+2}, v_{\Delta+3}\right),\left(v_{\Delta+4}, v_{\Delta+5}\right)$, $\ldots,\left(v_{h-2}, v_{h-1}\right)$, and $\left(v_{h}, v_{1}\right)$ (Recall that the degree of $v_{1}$ is odd, and hence, at least 1). Since every vertex of $G_{h}^{\Delta}$ has even degree, $G_{h}^{\Delta}$ has an Eulerian closed trail. Hence, $K_{h}^{\Delta}$ has the trail of length $\left(\begin{array}{l}h \\ 2\end{array}\right)-\Delta-\frac{h-\Delta}{2}$.

This completes the proof of the "if" part of Claim 8.

Proof of the "only-if" part of Claim 8. We distinguish two cases.

Case $1 h$ is odd: If $\Delta$ is odd [resp., even], $K_{h}^{\Delta}$ has $\Delta+1$ [resp., $\Delta$ ] odd vertices. Since $v_{2}$ is an odd vertex, we have from Theorem $\mathrm{C}$ that $v_{2}$ and one more vertex can be remained odd vertices in the subgraph of $K_{h}^{\Delta}$ that has an Eulerian trail starting at $v_{2}$, and all the other odd vertices must be made into even vertices. Hence, at least $\frac{(\Delta+1)-2}{2}$ [resp., $\left.\frac{\Delta-2}{2}\right]$ edges must be deleted from $K_{h}^{\Delta}$ to obtain the subgraph, and it has at most $\left(\begin{array}{l}h \\ 2\end{array}\right)-\Delta-\frac{\Delta-1}{2}$ [resp., $\left.\left(\begin{array}{l}h \\ 2\end{array}\right)-\Delta-\frac{\Delta-2}{2}\right]$ edges.

Case $2 h$ is even: If $\Delta$ is odd [resp., even], $K_{h}^{\Delta}$ has $h-\Delta-1$ [resp., $h-\Delta$ ] odd vertices. Since $v_{2}$ is even vertex, we have from Theorem $C$ that all the odd vertices must be made into even vertices in the subgraph of $K_{h}^{\Delta}$ that has an Eulerian trail starting at $v_{2}$. Hence, at least $\frac{h-\Delta-1}{2}$ [resp., $\frac{h-\Delta}{2}$ ] edges must be deleted from $K_{h}^{\Delta}$ to obtain the subgraph, and it has at most $\left(\begin{array}{l}h \\ 2\end{array}\right)-\Delta-\frac{h-\Delta-1}{2}\left[\right.$ resp., $\left.\left(\begin{array}{l}h \\ 2\end{array}\right)-\Delta-\frac{h-\Delta}{2}\right]$ edges.

This completes the proof of the "only-if" part of Claim 8.
Now, we complete the proof of Lemma 7.

\section{Proof of Theorem 2}

Let $P_{t}$ be the longest path of $C_{t, \Delta}$, and let $h=h\left(P_{t}\right)$. We can prove the following by arguments similar to Lemmas 4,5 , and 6.

Lemma 9.

- If $\Delta \geq h$, then $h\left(C_{t, \Delta}\right)=\Delta+1$.

- If $\Delta<h$, then $h\left(C_{t, \Delta}\right) \leq h+1$.

- $h\left(C_{t, \Delta}\right) \geq h$.

The following lemma completes the proof of Theorem 2. The rest of the section is devoted to the proof of the lemma.

Lemma 10. Suppose that $\Delta<h . C_{t, \Delta}$ can be colored harmoniously with $h$ colors if and only if

- $m \leq\left(\begin{array}{l}h \\ 2\end{array}\right)-\frac{\Delta-3}{2}$ when $h$ is odd and $\Delta$ is odd,

- $m \leq\left(\begin{array}{l}h \\ 2\end{array}\right)-\frac{\Delta-4}{2}$ when $h$ is odd and $\Delta$ is even,

- $m \leq\left(\begin{array}{l}h \\ 2\end{array}\right)-\frac{h-\Delta-1}{2}$ when $h$ is even and $\Delta$ is odd, and

$-m \leq\left(\begin{array}{l}h \\ 2\end{array}\right)-\frac{h-\Delta}{2}$ when $h$ is even and $\Delta$ is even.

Proof. As in the proof of Lemma 7, we assume w.l.o.g. that color 1 is assigned to the degree- $\Delta$ vertex, and colors $2,3, \ldots, \Delta+1$ are assigned to the vertices adjacent to it. See Fig. 2 (b) for example. Then, $t-3$ vertices remains uncolored, and they induce two paths of $C_{t, \Delta}$ (Note that one vertex can be regarded as a path). To prove Lemma 10, it suffices to show that the remaining paths can be colored harmoniously with $h$ colors if and only if the number of edges of $C_{t, \Delta}$ satisfies the inequality in the lemma. We assume w.l.o.g. that the end-vertex of the path is adjacent to the vertex with color 2 , and the end-vertex of the other path is adjacent to the vertex with color 3 (See Fig. 2 (b)).

We use the graph $K_{h}^{\Delta}$ defined in Sect.3. Recall that $V\left(K_{h}^{\Delta}\right)=\left\{v_{1}, v_{2}, \ldots, v_{h}\right\}$ and that each $v_{i}$ has color $i$. Notice that the edges of $K_{h}^{\Delta}$ denote the pair of colors which we can use for the harmonious coloring of the remaining paths, and the non-edges of $K_{h}^{\Delta}$ denote the pair of colors used for the harmonious coloring of the star $K_{1, \Delta}$ in $C_{t, \Delta}$. Coloring the remaining paths of $C_{t, \Delta}$ harmoniously is equivalent to obtaining a pair of edge-disjoint trails $W_{1}$ and $W_{2}$ in $K_{h}^{\Delta}$ such that $W_{1}$ starts at $v_{2}, W_{2}$ starts at $v_{3}$, and the sum of the length of $W_{1}$ and $W_{2}$ is $t-3$ (Notice that $W_{1}$ and $W_{2}$ must have length at least 1; otherwise the graph is not a comet but a shooting star). For example, $K_{5}^{4}$ in Fig. 3 has a pair of edge-disjoint trails $\left(v_{2}, a, v_{3}, b, v_{4}, c, v_{5}, d, v_{2}, e, v_{4}\right)$ and $\left(v_{3}, f, v_{5}\right)$, the sum of the length of which is 6 . As shown in Fig. 2 (b), the remaining paths of $C_{9,4}$ can be colored harmoniously according to the pair of trails. Then, the following claim proves Lemma 10 (Recall that $\left|E\left(K_{h}^{\Delta}\right)\right|=\left(\begin{array}{l}h \\ 2\end{array}\right)-\Delta$ ).

Claim 11. $K_{h}^{\Delta}$ has a pair of edge-disjoint trails $W_{1}$ and $W_{2}$ 


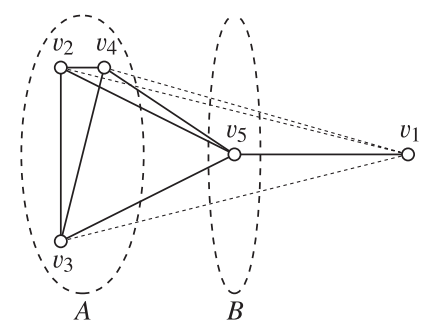

(a) Graph $K_{5}^{3}$.

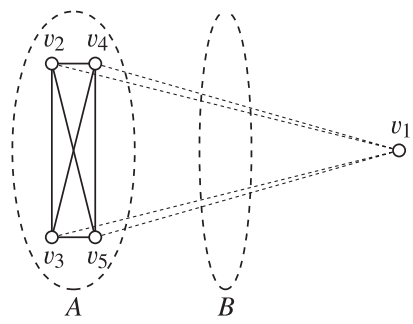

(b) Graph $K_{5}^{4}$.

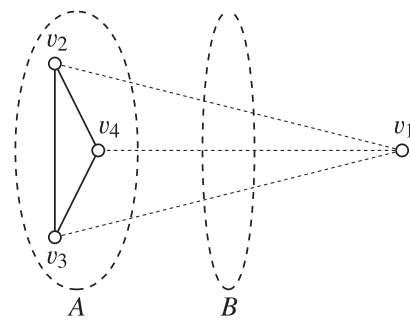

(c) Graph $K_{4}^{3}$

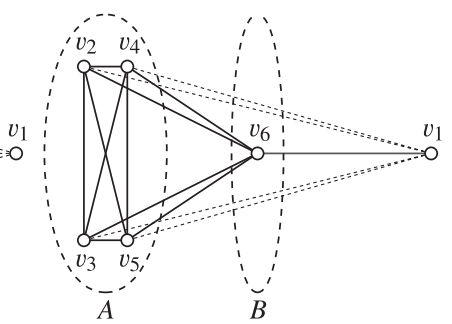

(d) Graph $K_{6}^{4}$.

Fig. 4 Graphs $K_{5}^{3}, K_{5}^{4}, K_{4}^{3}$, and $K_{6}^{4}$ with tripartition $\left(A, B, v_{1}\right)$. Dotted lines denote the edges deleted from the complete graphs. Edge $\left(v_{6}, v_{1}\right)$ of $K_{6}^{4}$ is not used in the trails.

such that

- $W_{1}$ starts at $v_{2}$ and has length $l_{1}$, and

- $W_{2}$ starts at $v_{3}$ and has length $l_{2}$,

if and only if $l_{1}$ and $l_{2}$ are any positive integers such that $l_{1}+l_{2}$ is at most

- $\left(\begin{array}{l}h \\ 2\end{array}\right)-\Delta-\frac{\Delta-3}{2}$ when $h$ is odd and $\Delta$ is odd,

- $\left(\begin{array}{l}h \\ 2\end{array}\right)-\Delta-\frac{\Delta-4}{2}$ when $h$ is odd and $\Delta$ is even,

- $\left(\begin{array}{l}h \\ 2\end{array}\right)-\Delta-\frac{h-\Delta-1}{2}$ when $h$ is even and $\Delta$ is odd, and

- $\left(\begin{array}{l}h \\ 2\end{array}\right)-\Delta-\frac{h-\Delta}{2}$ when $h$ is even and $\Delta$ is even.

Proof of the "if" part of Claim 11. We employ the arguments similar to those used in [13]. Let $K_{2, r}(r \geq 2)$ be the complete bipartite graph with bipartition $(U, V)$ such that $|U|=2$ and $|V|=r$. Let $G_{r}$ be the graph obtained from $K_{2, r}$ by adding an edge between the vertices in $U$. A graph $G$ is said to be partitioned into a pair of trails $W_{1}$ and $W_{2}$ if $E(G)=E\left(W_{1}\right) \cup E\left(W_{2}\right)$ and $E\left(W_{1}\right) \cap E\left(W_{2}\right)=\emptyset$.

(i). Let $r$ be a positive even integer, and let $r_{1}$ and $r_{2}$ be non-negative even integers with $r_{1}+r_{2}=r . K_{2, r}$ can be partitioned into two closed trails $W_{1}$ and $W_{2}$ such that $\left|E\left(W_{1}\right)\right|=2 r_{1}$ and $\left|E\left(W_{2}\right)\right|=2 r_{2}$ (Notice that $\left|E\left(W_{1}\right)\right|$ or $\left|E\left(W_{2}\right)\right|$ can be 0$)$.

Proof of $(i)$. Let $V_{1}$ be a set of $r_{1}$ vertices in $V$, and let $V_{2}$ be the set of other $r_{2}$ vertices in $V$. We have from Theorem $\mathrm{C}$ that the subgraph $K_{2, r_{1}}$ of $K_{2, r}$ induced by $U \cup V_{1}$ has an Eulerian closed trail, since every vertex of $K_{2, r_{1}}$ has even degree. Similarly, the subgraph $K_{2, r_{2}}$ of $K_{2, r}$ induced by $U \cup$ $V_{2}$ has an Eulerian closed trail. Since any edge of $K_{2, r}$ is in either $K_{2, r_{1}}$ or $K_{2, r_{2}}$, we have (i).

(ii). Let $r$ be a positive odd integer, and let $r_{1}$ and $r_{2}$ be nonnegative integers with $r_{1}+r_{2}=r$. We assume that $r_{1}$ is odd and $r_{2}$ is even. $G_{r}$ can be partitioned into two closed trails $W_{1}$ and $W_{2}$ such that $\left|E\left(W_{1}\right)\right|=2 r_{1}+1$ and $\left|E\left(W_{2}\right)\right|=2 r_{2}$ (Notice that $\left|E\left(W_{1}\right)\right| \geq 3$ while $\left|E\left(W_{2}\right)\right|$ can be 0 ).

Proof of (ii). Let $V_{1}$ be a set of $r_{1}$ vertices in $V$, and let $V_{2}$ be the set of other $r_{2}$ vertices in $V$. We have from Theorem $\mathrm{C}$ that the subgraph $G_{r_{1}}$ of $G_{r}$ induced by $U \cup V_{1}$ has an Eulerian closed trail, since every vertex of $G_{r_{1}}$ has even degree. Similarly, the subgraph $K_{2, r_{2}}$ of $G_{r}$ induced by the edges between $U$ and $V_{2}$ has an Eulerian closed trail. Since any edge of $G_{r}$ is in either $G_{r_{1}}$ or $K_{2, r_{2}}$, we have (ii).

Now, we are ready to prove the "if" part of Claim 11. Recall that $A\left(K_{h}^{\Delta}\right)=\left\{v_{2}, v_{3}, \ldots, v_{\Delta+1}\right\}$ and $B\left(K_{h}^{\Delta}\right)=\left\{v_{\Delta+2}\right.$, $\left.v_{\Delta+3}, \ldots, v_{h}\right\}$. We prove by induction on $\left|A\left(K_{h}^{\Delta}\right)\right|$ and $\left|B\left(K_{h}^{\Delta}\right)\right|$. Since $\left|A\left(K_{h}^{\Delta}\right)\right|=\Delta$ and $\left|B\left(K_{h}^{\Delta}\right)\right|=h-\Delta-1$, we have the following:

$$
\begin{array}{rlrl}
\left|A\left(K_{h+2}^{\Delta+2}\right)\right| & =\left|A\left(K_{h}^{\Delta}\right)\right|+2 ; & & \left|B\left(K_{h+2}^{\Delta+2}\right)\right|=\left|B\left(K_{h}^{\Delta}\right)\right| ; \\
\left|A\left(K_{h+2}^{\Delta}\right)\right|=\left|A\left(K_{h}^{\Delta}\right)\right| ; & & \left|B\left(K_{h+2}^{\Delta}\right)\right|=\left|B\left(K_{h}^{\Delta}\right)\right|+2 .
\end{array}
$$

We take the pair of trails from $K_{h+2}^{\Delta+2}$ and from $K_{h+2}^{\Delta}$ with the pair of trails in $K_{h}^{\Delta}$. We distinguish two cases.

Case $1 h$ is odd: Suppose that $\Delta$ is odd [resp., even]. Recall that $3 \leq \Delta<h$. In the base case, it can be verified that $K_{5}^{3}$ [resp., $\left.K_{5}^{4}\right]$ has the pair of trails, one of which starts at $v_{2}$ and the other starts at $v_{3}$, such that the length of the trails are any positive integers whose sum is $\left(\begin{array}{l}5 \\ 2\end{array}\right)-3-\frac{3-3}{2}=7$ [resp., $\left(\begin{array}{l}5 \\ 2\end{array}\right)-4-\frac{4-4}{2}=6$ ]. See Fig. 4 (a) [resp., Fig. 4 (b)].

Assume by induction that for any positive integers $l_{1}$ and $l_{2}$ such that $l_{1}+l_{2}=\left(\begin{array}{l}h \\ 2\end{array}\right)-\Delta-\frac{\Delta-3}{2}$ [resp., $\left.\left(\begin{array}{l}h \\ 2\end{array}\right)-\Delta-\frac{\Delta-4}{2}\right]$, $K_{h}^{\Delta}$ has a pair of edge-disjoint trails $W_{1}$ and $W_{2}$ such that $W_{1}$ starts at $v_{2}$ and has length $l_{1}$, and $W_{2}$ starts at $v_{3}$ and has length $l_{2}$.

We first show that for any positive integers $l_{1}$ and $l_{2}$ such that $l_{1}+l_{2}=\left(\begin{array}{c}h+2 \\ 2\end{array}\right)-(\Delta+2)-\frac{(\Delta+2)-3}{2}$ [resp., $\left(\begin{array}{c}h+2 \\ 2\end{array}\right)-(\Delta+$ $\left.2)-\frac{(\Delta+2)-4}{2}\right], K_{h+2}^{\Delta+2}$ has a pair of edge-disjoint trails $W_{1}$ and $W_{2}$ such that $W_{1}$ starts at $v_{2}$ and has length $l_{1}$, and $W_{2}$ starts at $v_{3}$ and has length $l_{2}$. The subgraph $I$ of $K_{h+2}^{\Delta+2}$ induced by $\left\{v_{1}, v_{2}, \ldots, v_{\Delta+1}, v_{\Delta+4}, v_{\Delta+5}, \ldots, v_{h+2}\right\}$ is isomorphic to $K_{h}^{\Delta}$. Since $v_{\Delta+2}$ and $v_{\Delta+3}$ are adjacent to every vertex in $V(I) \backslash\left\{v_{1}\right\}$, the subgraph $J=K_{h+2}^{\Delta+2}-E(I)-\left(v_{\Delta+2}, v_{\Delta+3}\right)-v_{1}$ is isomorphic to $K_{2, h-1}$. Since $h-1$ is even, we have from (i) that $J$ can be partitioned into two closed trails whose length are $2 r_{1}$ and $2 r_{2}$, respectively. Here, $r_{1}$ and $r_{2}$ are non-negative even integers such that $r_{1}+r_{2}=h-1,2 r_{1}<l_{1}$, and $2 r_{2}<l_{2}$. We can assume w.l.o.g. that the closed trail of length $2 r_{1}$ has $v_{2}$ and the closed trail of length $2 r_{2}$ has $v_{3}$. Let $l_{1}^{\prime}=l_{1}-2 r_{1}$ and $l_{2}^{\prime}=l_{2}-2 r_{2}$. It is straightforward to see that $l_{1}^{\prime}+l_{2}^{\prime}=$ $\left(\begin{array}{l}h \\ 2\end{array}\right)-\Delta-\frac{\Delta-3}{2}$ [resp., $\left(\begin{array}{l}h \\ 2\end{array}\right)-\Delta-\frac{\Delta-4}{2}$ ]. By assumption, $I$ has a pair of edge-disjoint trails $W_{1}^{\prime}$ and $W_{2}^{\prime}$ such that $W_{1}^{\prime}$ starts 
at $v_{2}$ and has length $l_{1}^{\prime}$, and $W_{2}^{\prime}$ starts at $v_{3}$ and has length $l_{2}^{\prime}$. We can obtain the two trails $W_{1}$ and $W_{2}$ of $K_{h+2}^{\Delta+2}$ as follows: $W_{1}$ starts at $v_{2}$, traverses $2 r_{1}$ edges of the closed trail in $J$, and traverses $l_{1}^{\prime}$ edges in $W_{1}^{\prime} ; W_{2}$ starts at $v_{3}$, traverses $2 r_{2}$ edges of the closed trail in $J$, and traverses $l_{2}^{\prime}$ edges in $W_{2}^{\prime}$.

We next show that for any positive integers $l_{1}$ and $l_{2}$ such that $l_{1}+l_{2}=\left(\begin{array}{c}h+2 \\ 2\end{array}\right)-\Delta-\frac{\Delta-3}{2}$ [resp., $\left(\begin{array}{c}h+2 \\ 2\end{array}\right)-\Delta-\frac{\Delta-4}{2}$ ], $K_{h+2}^{\Delta}$ has a pair of edge-disjoint trails $W_{1}$ and $W_{2}$ such that $W_{1}$ starts at $v_{2}$ and has length $l_{1}$, and $W_{2}$ starts at $v_{3}$ and has length $l_{2}$. We assume w.l.o.g. that $l_{1} \geq l_{2}$. Since $l_{1}+$ $l_{2} \geq 8$ for any $h$ and $\Delta$, we have $l_{1} \geq 4$. The subgraph $I$ of $K_{h+2}^{\Delta}$ induced by $\left\{v_{1}, v_{2}, \ldots, v_{h}\right\}$ is isomorphic to $K_{h}^{\Delta}$. Since $v_{h+1}$ and $v_{h+2}$ are adjacent to every vertex in $I$, the subgraph $J=K_{h+2}^{\Delta}-E(I)$ is isomorphic to $G_{h}$. Since $h$ is odd, we have from (ii) that $J$ can be partitioned into two closed trails whose length are $2 r_{1}+1$ and $2 r_{2}$, respectively. Here, $r_{1}$ is a non-negative odd integer and $r_{2}$ is a non-negative even integer such that $r_{1}+r_{2}=h, 2 r_{1}+1<l_{1}$, and $2 r_{2}<l_{2}$. We can assume w.l.o.g. that the closed trail of length $2 r_{1}+1$ has $v_{2}$ and the closed trail of length $2 r_{2}$ has $v_{3}$. Let $l_{1}^{\prime}=l_{1}-2 r_{1}-1$ (recall that $2 r_{1}+1 \geq 3$ but $l_{1} \geq 4$ ) and $l_{2}^{\prime}=l_{2}-2 r_{2}$. It is straightforward to see that $l_{1}^{\prime}+l_{2}^{\prime}=\left(\begin{array}{l}h \\ 2\end{array}\right)-\Delta-\frac{\Delta-3}{2}$ [resp., $\left.\left(\begin{array}{l}h \\ 2\end{array}\right)-\Delta-\frac{\Delta-4}{2}\right]$. By assumption, $I$ has a pair of edge-disjoint trails $W_{1}^{\prime}$ and $W_{2}^{\prime}$ such that $W_{1}^{\prime}$ starts at $v_{2}$ and has length $l_{1}^{\prime}$, and $W_{2}^{\prime}$ starts at $v_{3}$ and has length $l_{2}^{\prime}$. We can obtain the two trails $W_{1}$ and $W_{2}$ of $K_{h+2}^{\Delta}$ as follows: $W_{1}$ starts at $v_{2}$, traverses $2 r_{1}+1$ edges of the closed trail in $J$, and traverses $l_{1}^{\prime}$ edges in $W_{1}^{\prime}$; $W_{2}$ starts at $v_{3}$, traverses $2 r_{2}$ edges of the closed trail in $J$, and traverses $l_{2}^{\prime}$ edges in $W_{2}^{\prime}$.

Case $2 h$ is even: Suppose that $\Delta$ is odd [resp., even]. Recall that $3 \leq \Delta<h$. In the base case, it can be verified that $K_{4}^{3}$ [resp., $K_{6}^{4}$ ] has the pair of trails, one of which starts at $v_{2}$ and the other starts at $v_{3}$, such that the length of the trails are any positive integers whose sum is $\left(\begin{array}{l}4 \\ 2\end{array}\right)-3-\frac{4-3-1}{2}=3$ [resp., $\left(\begin{array}{l}6 \\ 2\end{array}\right)-4-\frac{6-4}{2}=10$ ]. See Fig. 4 (c) [resp., Fig. 4 (d)].

Assume by induction that for any positive integers $l_{1}$ and $l_{2}$ such that $l_{1}+l_{2}=\left(\begin{array}{l}h \\ 2\end{array}\right)-\Delta-\frac{h-\Delta-1}{2}\left[\right.$ resp., $\left.\left(\begin{array}{l}h \\ 2\end{array}\right)-\Delta-\frac{h-\Delta}{2}\right]$, $K_{h}^{\Delta}$ has a pair of edge-disjoint trails $W_{1}$ and $W_{2}$ such that $W_{1}$ starts at $v_{2}$ and has length $l_{1}$, and $W_{2}$ starts at $v_{3}$ and has length $l_{2}$.

We first show that for any positive integers $l_{1}$ and $l_{2}$ such that $l_{1}+l_{2}=\left(\begin{array}{c}h+2 \\ 2\end{array}\right)-(\Delta+2)-\frac{(h+2)-(\Delta+2)-1}{2}$ [resp., $\left.\left(\begin{array}{c}h+2 \\ 2\end{array}\right)-(\Delta+2)-\frac{(h+2)-(\Delta+2)}{2}\right], K_{h+2}^{\Delta+2}$ has a pair of edgedisjoint trails $W_{1}$ and $W_{2}$ such that $W_{1}$ starts at $v_{2}$ and has length $l_{1}$, and $W_{2}$ starts at $v_{3}$ and has length $l_{2}$. We assume w.l.o.g. that $l_{1} \geq l_{2}$. Since $l_{1}+l_{2} \geq 8$ for any $h$ and $\Delta$, we have $l_{1} \geq 4$. The subgraph $I$ of $K_{h+2}^{\Delta+2}$ induced by $\left\{v_{1}, v_{2}, \ldots, v_{\Delta+1}, v_{\Delta+4}, v_{\Delta+5}, \ldots, v_{h+2}\right\}$ is isomorphic to $K_{h}^{\Delta}$. Since $v_{\Delta+2}$ and $v_{\Delta+3}$ are adjacent to every vertex in $V(I) \backslash\left\{v_{1}\right\}$, the subgraph $J=K_{h+2}^{\Delta+2}-E(I)-v_{1}$ is isomorphic to $G_{h-1}$. Since $h-1$ is odd, we have from (ii) that $J$ can be partitioned into two closed trails whose length are $2 r_{1}+1$ and $2 r_{2}$, respectively. Here, $r_{1}$ is a non-negative odd integer and $r_{2}$ is a non-negative even integer such that $r_{1}+r_{2}=h-1$,
$2 r_{1}+1<l_{1}$, and $2 r_{2}<l_{2}$. We can assume w.l.o.g. that the closed trail of length $2 r_{1}+1$ has $v_{2}$ and the closed trail of length $2 r_{2}$ has $v_{3}$. Let $l_{1}^{\prime}=l_{1}-2 r_{1}-1$ (recall that $2 r_{1}+1 \geq 3$ but $l_{1} \geq 4$ ) and $l_{2}^{\prime}=l_{2}-2 r_{2}$. It is straightforward to see that $l_{1}^{\prime}+l_{2}^{\prime}=\left(\begin{array}{l}h \\ 2\end{array}\right)-\Delta-\frac{h-\Delta-1}{2}$ [resp., $\left(\begin{array}{l}h \\ 2\end{array}\right)-\Delta-\frac{h-\Delta}{2}$ ]. By assumption, $I$ has a pair of edge-disjoint trails $W_{1}^{\prime}$ and $W_{2}^{\prime}$ such that $W_{1}^{\prime}$ starts at $v_{2}$ and has length $l_{1}^{\prime}$, and $W_{2}^{\prime}$ starts at $v_{3}$ and has length $l_{2}^{\prime}$. We can obtain the two trails $W_{1}$ and $W_{2}$ of $K_{h+2}^{\Delta+2}$ as follows: $W_{1}$ starts at $v_{2}$, traverses $2 r_{1}+1$ edges of the closed trail in $J$, and traverses $l_{1}^{\prime}$ edges in $W_{1}^{\prime} ; W_{2}$ starts at $v_{3}$, traverses $2 r_{2}$ edges of the closed trail in $J$, and traverses $l_{2}^{\prime}$ edges in $W_{2}^{\prime}$.

We next show that for any positive integers $l_{1}$ and $l_{2}$ such that $l_{1}+l_{2}=\left(\begin{array}{c}h+2 \\ 2\end{array}\right)-\Delta-\frac{(h+2)-\Delta-1}{2}$ [resp., $\left(\begin{array}{c}h+2 \\ 2\end{array}\right)-\Delta-$ $\frac{(h+2)-\Delta}{2}$ ], $K_{h+2}^{\Delta}$ has a pair of edge-disjoint trails $W_{1}$ and $W_{2}$ such that $W_{1}$ starts at $v_{2}$ and has length $l_{1}$, and $W_{2}$ starts at $v_{3}$ and has length $l_{2}$. The subgraph $I$ of $K_{h+2}^{\Delta}$ induced by $\left\{v_{1}, v_{2}, \ldots, v_{h}\right\}$ is isomorphic to $K_{h}^{\Delta}$. Since $v_{h+1}$ and $v_{h+2}$ are adjacent to every vertex in $I$, the subgraph $J=K_{h+2}^{\Delta}-$ $E(I)-\left(v_{h+1}, v_{h+2}\right)$ is isomorphic to $K_{2, h}$. Since $h$ is even, we have from (i) that $J$ can be partitioned into two closed trails whose length are $2 r_{1}$ and $2 r_{2}$, respectively. Here, $r_{1}$ and $r_{2}$ are non-negative even integers such that $r_{1}+r_{2}=h, 2 r_{1}<l_{1}$, and $2 r_{2}<l_{2}$. We can assume w.l.o.g. that the closed trail of length $2 r_{1}$ has $v_{2}$ and the closed trail of length $2 r_{2}$ has $v_{3}$. Let $l_{1}^{\prime}=l_{1}-2 r_{1}$ and $l_{2}^{\prime}=l_{2}-2 r_{2}$. It is straightforward to see that $l_{1}^{\prime}+l_{2}^{\prime}=\left(\begin{array}{l}h \\ 2\end{array}\right)-\Delta-\frac{h-\Delta-1}{2}$ [resp., $\left(\begin{array}{l}h \\ 2\end{array}\right)-\Delta-\frac{h-\Delta}{2}$ ]. By assumption, $I$ has a pair of edge-disjoint trails $W_{1}^{\prime}$ and $W_{2}^{\prime}$ such that $W_{1}^{\prime}$ starts at $v_{2}$ and has length $l_{1}^{\prime}$, and $W_{2}^{\prime}$ starts at $v_{3}$ and has length $l_{2}^{\prime}$. We can obtain the two trails $W_{1}$ and $W_{2}$ of $K_{h+2}^{\Delta}$ as follows: $W_{1}$ starts at $v_{2}$, traverses $2 r_{1}$ edges of the closed trail in $J$, and traverses $l_{1}^{\prime}$ edges in $W_{1}^{\prime} ; W_{2}$ starts at $v_{3}$, traverses $2 r_{2}$ edges of the closed trail in $J$, and traverses $l_{2}^{\prime}$ edges in $W_{2}^{\prime}$.

This completes the proof of the "if" part of Claim 11.

Proof of the "only-if" part of Claim 11. Recall that a graph $G$ is said to be partitioned into a pair of trails $W_{1}$ and $W_{2}$ if $E(G)=E\left(W_{1}\right) \cup E\left(W_{2}\right)$ and $E\left(W_{1}\right) \cap E\left(W_{2}\right)=\emptyset$. It is easy to see the following.

Claim 12. If a graph $G$ can be partitioned into a pair of trails, $G$ has at most four odd vertices. Moreover, if the trails start at distinct even vertices, $G$ has no odd vertices.

Let $G_{h}^{\Delta}$ be a subgraph of $K_{h}^{\Delta}$ that can be partitioned into the pair of trails, one of which starts at $v_{2}$ and the other starts at $v_{3}$. We distinguish two cases.

Case $1 h$ is odd: If $\Delta$ is odd [resp., even], $K_{h}^{\Delta}$ has $\Delta+1$ [resp., $\Delta$ ] odd vertices. Since $v_{2}$ and $v_{3}$ have odd degree, we have from Claim 12 that four odd vertices including $v_{2}$ and $v_{3}$ can be remained odd vertices in $G_{h}^{\Delta}$, and all the other odd vertices must be made into even vertices. Hence, at least $\frac{(\Delta+1)-4}{2}$ [resp., $\frac{\Delta-4}{2}$ ] edges must be deleted from $K_{h}^{\Delta}$ to obtain $G_{h}^{\Delta}$, and $G_{h}^{\Delta}$ has at most $\left(\begin{array}{l}h \\ 2\end{array}\right)-\Delta-\frac{\Delta-3}{2}\left[\right.$ resp., $\left.\left(\begin{array}{l}h \\ 2\end{array}\right)-\Delta-\frac{\Delta-4}{2}\right]$ 
edges.

Case $2 h$ is even: If $\Delta$ is odd [resp., even], $K_{h}^{\Delta}$ has $h-\Delta-1$ [resp., $h-\Delta$ ] odd vertices. Notice that $h-\Delta-1$ [resp., $h-\Delta$ ] is even. Since $v_{2}$ and $v_{3}$ have even degree, we have from Claim 12 that all the odd vertices must be made into even vertices in $G_{h}^{\Delta}$. Hence, at least $\frac{h-\Delta-1}{2}$ [resp., $\frac{h-\Delta}{2}$ ] edges must be deleted from $K_{h}^{\Delta}$ to obtain $G_{h}^{\Delta}$, and $G_{h}^{\Delta}$ has at $\operatorname{most}\left(\begin{array}{l}h \\ 2\end{array}\right)-\Delta-\frac{h-\Delta-1}{2}\left[\right.$ resp., $\left.\left(\begin{array}{l}h \\ 2\end{array}\right)-\Delta-\frac{h-\Delta}{2}\right]$ edges.

This completes the proof of the "only-if" part of Claim 11.

Now, we complete the proof of Lemma 10.

\section{Proof of Theorem 3}

Let $T_{t}$ be a 3-regular caterpillar with the longest path on $t$ vertices, let $P_{t}$ be the longest path of $T_{t}$, and let $h=h\left(P_{t}\right)$. We show in this section that $T_{t}$ can be colored harmoniously with $h+\lfloor(h-1) / 2\rfloor$ colors. We first color the vertices on $P_{t}$ harmoniously with $h$ colors, then color the remaining vertices with $\lfloor(h-1) / 2\rfloor$ colors. See Fig. 2 (c) for example of such a coloring of a 3-regular caterpillar.

Now, it suffices to show that in any harmonious coloring of $P_{t}$, at most $\lfloor(h-1) / 2\rfloor$ inner vertices have the same color. Suppose contrary that at least $\lfloor(h-1) / 2\rfloor+1$ inner vertices have, w.l.o.g., color $h$. Let $V_{h}$ be the set of such inner vertices. Let $N\left(V_{h}\right)$ be the set of vertices on $P_{t}$ adjacent to a vertex in $V_{h}$. Since the distance between any pair of vertices in $V_{h}$ is at least 3 , we have $\left|N\left(V_{h}\right)\right|=2\left|V_{h}\right|$. Since the vertices on $P_{t}$ are colored harmoniously, the vertices in $N\left(V_{h}\right)$ must have different colors from colors $1,2, \ldots, h-1$. However, since $(h-1) / 2-1<\lfloor(h-1) / 2\rfloor$, we have that $h-1<2(\lfloor(h-1) / 2\rfloor+1) \leq\left|N\left(V_{h}\right)\right|$, a contradiction. Hence, at most $\lfloor(h-1) / 2\rfloor$ inner vertices on $P_{t}$ have the same color, and we have Theorem 3.

\section{Concluding Remarks}

This paper showed in Theorems 1 and 2 the harmonious chromatic number of a shooting star and a comet, respectively. We also showed in Theorem 3 the upper bound of the harmonious chromatic number of a 3-regular caterpillar.

Akbari et al. [2] show that for any forest $T, h(T)=$ $\Delta(T)+1$ if $\Delta(T) \geq \frac{n+2}{3}$ and $T$ has no pair of non-adjacent vertices of degree $\Delta(T)$. Here, $n=|V(T)|$ and $\Delta(T)$ is the maximum degree of $T$. They also show that the bound $\Delta(T) \geq$ $\frac{n+2}{3}$ is sharp, that is, for any integer $d \geq 3$ they present a caterpillar $T_{d}$ such that (i) $\Delta\left(T_{d}\right)=d$, (ii) $T_{d}$ has no pair of non-adjacent vertices of degree $d$, (iii) $\left|V\left(T_{d}\right)\right|=3 d-1$, and (iv) $h\left(T_{d}\right) \geq d+2$. In the case of shooting stars and comets (that is, caterpillars with at most one vertex of degree more than 2), Theorems 1 and 2 state that $h\left(T^{\prime}\right)=\Delta\left(T^{\prime}\right)+1$ if $\Delta\left(T^{\prime}\right) \geq h\left(P^{\prime}\right)$ for any such caterpillar $T^{\prime}$, where $P^{\prime}$ is the longest path of $T^{\prime}$. The theorems also imply that the bound $\Delta\left(T^{\prime}\right) \geq h\left(P^{\prime}\right)$ is sharp, that is, for any integer $d \geq 3$ we have a caterpillar $T_{d}^{\prime}$ with at most one vertex of degree more than 2 such that (i) $\Delta\left(T_{d}^{\prime}\right)=d$, (ii) $\Delta\left(T_{d}^{\prime}\right)<h\left(P_{d}^{\prime}\right)$, and (iii) $h\left(T_{d}^{\prime}\right) \geq d+2$. The bound $\Delta\left(T^{\prime}\right) \geq h\left(P^{\prime}\right)$ is significantly smaller than the general bound $\Delta\left(T^{\prime}\right) \geq \frac{n+2}{3}$, since $h\left(P^{\prime}\right)=O(\sqrt{n})($ Recall that $k(G)=\lceil(1+\sqrt{8 m+1}) / 2\rceil$ for any graph $G$ with $m$ edges).

We also note that Edwards [10] conjectures that for any tree $T, h(T) \leq k(T)+\Delta(T)$. Theorems 1 and 2 imply that this conjecture holds for caterpillars with at most one vertex of degree more than 2. On the other hand, Theorem 3 does not imply the conjecture since 3-regular caterpillar $T_{315}$ is a counter example, that is, $h\left(P_{315}\right)+\left\lfloor\left(h\left(P_{315}\right)-1\right) / 2\right\rfloor>$ $k\left(T_{315}\right)+3$, where $T_{315}$ is the 3-regular caterpillar with the longest path on 315 vertices, and $P_{315}$ is the longest path of $T_{315}$. (Note that $k\left(T_{315}\right)=36$ and $h\left(P_{315}\right)=27$ ). Hence, it remains an open problem to ask whether $h(T) \leq k(T)+3$ for any 3-regular caterpillar $T$.

We finally note that the complexity of the harmonious coloring problem for general caterpillars also remains open.

\section{Acknowledgments}

We are grateful to anonymous referees for careful reading and helpful comments. The research was partially supported by JSPS KAKENHI Grant Number 26330007, and JSPS Grant-in-Aid for JSPS Fellows (26.8924).

\section{References}

[1] A. Aflaki, S. Akbari, K.J. Edwards, D.S. Eskandani, M. Jamaali, and H. Ravanbod, "On harmonious colouring of trees," Electr. J. Comb., vol.19, no.1, pp.1-9, 2012.

[2] S. Akbari, J. Kim, and A. Kostochka, "Harmonious coloring of trees with large maximum degree," Discrete Math., vol.312, no.10, pp.1633-1637, 2012.

[3] K. Asdre, K. Ioannidou, and S.D. Nikolopoulos, "The harmonious coloring problem is NP-complete for interval and permutation graphs," Discrete Appl. Math., vol.155, no.17, pp.2377-2382, 2007.

[4] K. Asdre and S.D. Nikolopoulos, "NP-completeness results for some problems on subclasses of bipartite and chordal graphs," Theor. Comput. Sci., vol.381, no.1-3, pp.248-259, 2007.

[5] H.L. Bodlaender, "Achromatic number is NP-complete for cographs and interval graphs," Inform. Process. Lett., vol.31, no.3, pp.135-138, 1989.

[6] J.A. Bondy and U.S.R. Murty, Graph Theory with Applications, Elsevier Science, 1976.

[7] N. Cairnie and K. Edwards, "Some results on the achromatic number," J. Graph Theory, vol.26, no.3, pp.129-136, 1997.

[8] D. Campbell and K. Edwards, "A new lower bound for the harmonious chromatic number," Australas. J. Combin., vol.29, pp.99-102, 2004.

[9] R.J. Cichelli, "Minimal perfect hash functions made simple," Commun. ACM, vol.23, no.1, pp.17-19, 1980.

[10] K. Edwards, "The harmonious chromatic number and the achromatic number," in Surveys in Combinatorics, 1997, ed. R.A. Bailey, London Mathematical Society Lecture Note Series, no.241, pp.1347, Cambridge University Press, 1997.

[11] K. Edwards and C. McDiarmid, "The complexity of harmonious colouring for trees," Discrete Appl. Math., vol.57, no.2-3, pp.133-144, 1995.

[12] O. Frank, F. Harary, and M. Plantholt, "The line-distinguishing chromatic number of a graph," Ars Combin., vol.14, pp.241-252, 1982.

[13] J.P. Georges, "On the harmonious coloring of collections of graphs," 
J. Graph Theory, vol.20, no.2, pp.241-254, 1995.

[14] J.E. Hppcroft and M.S. Krishnamoorthy, "On the harmonious coloring of graphs," SIAM J. Alg. Disc. Meth., vol.4, no.3, pp.306-311, 1983.

[15] K. Ioannidou and S.D. Nikolopoulos, "Harmonious coloring on subclasses of colinear graphs," Proc. 4th International Workshop on Algorithms and Computation (WALCOM), Lecture Notes in Comput. Sci., vol.5942, pp.136-148, 2010.

[16] M. Kubale, "Harmonious coloring of graphs," in Graph Colorings, ed. M. Kubale, Contemporary Mathematics, vol.352, ch.7, American Mathematical Society, 2004.

[17] Z. Miller and D. Pritikin, "The harmonious coloring number of a graph,” Discrete Math., vol.93, no.2-3, pp.211-228, 1991.

[18] J. Mitchem, "On the harmonious chromatic number of a graph," Discrete Math., vol.74, no.1-2, pp.151-157, 1989.

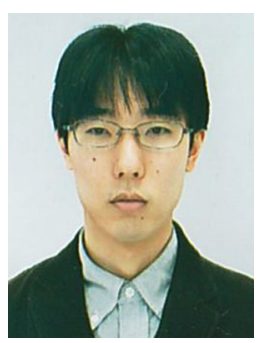

Asahi Takaoka received his B.E. degree in computer science and M.E. and D.E. degree in communications and integrated systems from Tokyo Institute of Technology, Tokyo, Japan, in 2010, 2012, and 2015, respectively. He has been a research fellow of Japan Society for the Promotion of Science (JSPS) from April 2014. He is now a postdoctoral researcher at Tokyo Institute of Technology. He is interested in algorithmic graph theory with applications. He is a member of IEEE and IEICE.

Shingo Okuma received his B.E. degree in computer science and M.E. degree in communications and integrated systems from Tokyo Institute of Technology, Tokyo, Japan, in 2012 and 2014, respectively.

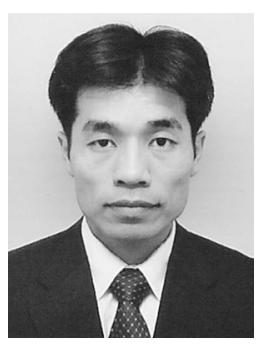

Satoshi Tayu received the B.E., M.E., and D.E. degrees in electrical and electronic engineering from Tokyo Institute of Technology, Tokyo, Japan, in 1992, 1994, and 1997, respectively. From 1997 to 2003, he was a research associate in the School of Information Science, Japan Advanced Institute of Science and Technology, Ishikawa, Japan. He is currently an assistant professor in the Department of Communications and Computer Engineering, Graduate School of Science and Engineering, Tokyo Institute of Technology. His research interests are in parallel computation. $\mathrm{He}$ is a member of IPSJ.

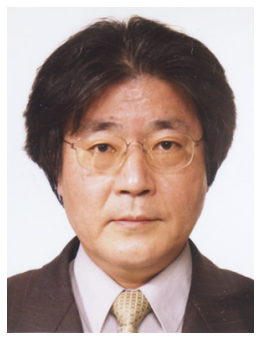

Shuichi Ueno received the B.E. degree in electronic engineering from Yamanashi University, Yamanashi, Japan, in 1976, and M.E. and D.E. degrees in electronic engineering from Tokyo Institute of Technology, Tokyo, Japan, in 1978 and 1982, respectively. Since 1982 he has been with Tokyo Institute of Technology, where he is now a professor in the Department of Communications and Computer Engineering, Graduate School of Science and Engineering. His research interests are in the theory of parallel and VLSI computation. He received the best paper award from the Institute of Electronics and Communication Engineers of Japan in 1986, the 30th anniversary best paper award from the Information Processing Society of Japan in 1990, and the best paper award of APCCAS 2000 from IEEE in 2000. Dr Ueno is a Fellow of IEICE, and a member of IEEE, SIAM, and IPSJ. 